\title{
Effect of Ethanol and of Noise on Reaction Time in the Monkey: Variation with Stimulus Level
}

\author{
David B. Moody ${ }^{1}$, Gail Winger ${ }^{2}$, James H. Woods ${ }^{2}$, and William C. Stebbins ${ }^{1}$ \\ ${ }^{1}$ Kresge Hearing Research Institute, University of Michigan Medical School, Ann Arbor, MI 48109, U.S.A. \\ 2 Department of Pharmacology, University of Michigan Medical School, Ann Arbor, MI 48109, U.S.A.
}

\begin{abstract}
To determine whether the latency-increasing effects of ethanol were differential with respect to the intensity of the stimulus that initiated the response, three rhesus monkeys were trained on a behavioral task in which the latency of a simple motor response was measured following the onset of a pure tone stimulus. Following training, the animals were tested at a number of different tone intensities and functions relating latency to tone intensity were constructed. When these were stable, the animals were given ethanol in doses of $1.0-2.5 \mathrm{~g} / \mathrm{kg}$ and the effects on response latencies to different tone intensities were determined. It was found that, for all except the lowest stimulus levels, the effect of ethanol was dose-related, while for a given dose the effect was equal across intensity. These results indicate that the effects of ethanol in this situation are on response execution rather than stimulus detection. The effects of ethanol were compared to those of exposure to high intensity noise. This treatment, which affects primarily the inner ear, resulted in substantial increases in latency to low intensity tones, but little, if any, shift at high intensities.
\end{abstract}

Key words: Alcohol - Ethanol - Reaction time Latency - Rhesus monkeys - Noise - Stimulus level - Stimulus intensity

Latency (reaction time) of an operant response has a long history as a dependent variable in experimental psychology. One use has been as an index of perceptual function with sensory stimuli presented at levels which are clearly detectable. Over a wide range of stimulus levels in a number of sensory modalities, latency is inversely related to stimulus level such that latency decreases in an orderly monotonic way as stimulus level

Offprint requests to: D. B. Moody increases. This relationship has been used to derive suprathreshold perceptual matching functions, including studies of visual luminosity (Pollack, 1968), brightness (Moody, 1969), and of the loudness of tones (Chocholle, 1940; Stebbins, 1966; Moody, 1970; Pfingst et al., 1975a). Manipulations that affect sensory systems, such as experimentally induced auditory pathology, have predictable effects on the function relating response latency to auditory stimulus intensity [the latency-intensity (LI) function], and have been used in conjunction with the latency variable to study the basis of psychoacoustic phenomena in animals (Moody, 1970, 1973; Pfingst et al., 1975a). In many cases, these effects are differential with respect to stimulus level; that is, they have substantial latency-increasing effects on responses to low level stimuli, but no effects on the response latencies to high level stimuli.

The latency variable has also been used as an indicator of the actions of pharmacological agents. Ethanol has received particular attention in this regard, frequently in attempts to ascertain whether an ethanolinduced increase in reaction time might account for the fact that ethanol impairs driving skills. The findings have shown that, while ethanol does produce an increase in simple reaction time (Carpenter, 1962), the doses required for this increment are much higher than those that impair driving. The work on ethanol and reaction time has since focused on more complex tasks, with the frequent conclusion being that ethanol disrupts or slows central processing of information (Moskowitz and Roth, 1971), specifically at the stage of response selection (Zunder, 1977; Moskowitz and Burns, 1976; Tharp et al., 1974). Although this work is very elegant and informative, it does not deal with the mechanism of ethanol's effects on simple reaction time where performance is slowed, but where the required central processing is minimal. In the case of simple reaction time, impairment following ethanol ingestion is most likely due to a decreased ability to detect the stimulus, a decreased ability to respond as rapidly, or 
an impairment of central processing. To investigate the ability of ethanol to impair stimulus detection, the present study tested the effect of ethanol on reaction time to different intensities of an auditory stimulus. To our knowledge, there have been no previous studies which attempt to determine whether the latencyincreasing effect of ethanol is differential with respect to the level of the stimulus that initiates the latency response.

The present experiment examined operant responding of rhesus monkeys in a behavioral situation in which the basic interest was in the latency of an operant response to various levels of acoustic stimulation. The paradigm has a number of common elements with a standard human reaction time experiment in that there is a 'ready' signal followed by the auditory stimulus and this reaction time is measured. The completed response sequence is followed by food presentation. It is possible with the present task to generate a set of reaction times to a large range of auditory intensities within a single session and, hence, to construct a function relating the. sound pressure level of the auditory stimulus to reaction time. This paradigm was used to make a series of observations of the effects of graded doses of IV ethanol and of various noise exposures upon reaction time to varying sound pressure levels of an auditory stimulus. Since both ethanol and noise exposure are known to slow reaction time, the central question of these observations was whether the latency-increasing effect of ethanol was dependent on the level of the acoustic stimulus and whether the two interventions had similar or different effects upon the reaction time variable.

\section{Materials and Methods}

Subjects. Two male (M41 and M42) and one female (M47) subadult rhesus monkeys (Macaca mulatta) with no previous experimental history were maintained on a $22-\mathrm{h}$ food deprivation schedule in which they received a total of $75-100 \mathrm{~g}$ food either during the experimental session in the form of $190 \mathrm{mg}$ Noyes monkey pellets or immediately following the session in the form of Purina Monkey Chow. They also received a fresh fruit supplement. Their weights were as follows: M41 at $5.3 \mathrm{~kg}$; M42 at $5.7 \mathrm{~kg}$; and M47 at $4.7 \mathrm{~kg}$.

The monkeys were surgically implanted with chronic indwelling venous catheters for ethanol administration (Deneau et al., 1969) and fitted with leather vests.

Apparatus. Before each experimental session, the monkeys were put into restraint chairs and then placed in a double-walled soundproof room (Industrial Acoustics, series 1200, model 60). Their heads were restrained and calibrated earphones (Permoflux, PDR 600 with MX41/AR cushions) were carefully placed over the external ears. Details of the chair, head restraint, and earphone mounting are described by Moody et al. (1970). A chute into which food pellets could be delivered was placed near the animal's mouth and a small light was mounted near the chute. A telegraph key was mounted at the far end of an 8 in. $(20 \mathrm{~cm}) \times 3$ in. $(7.5 \mathrm{~cm})$ diameter Plexiglas tube. The tube, designed to minimize variation in response topography, was mounted on the chair at waist level.
The auditory stimuli were generated by a Hewlett-Packard $(200 \mathrm{CD})$ oscillator. The sound pressure was controlled by a remotely programmable attenuator. The tones were gated by a tone switch, which had a rise and decay time of $5 \mathrm{~ms}$. Contingencies were arranged and data were recorded by solid-state logic and a digital computer (Digital Equipment Corp., PDP-8). Response latencies were recorded on punched paper tape for later analysis.

Sound exposures were carried out in a separate double-walled sound room which was specially modified to make it reverberant. This room was equipped with two Altec Voice of the Theater speaker systems driven by a McIntosh power amplifier. White noise was generated by a General Radio (model 1382) noise generator and band limited with Allison (model 2BR) variable filters. The sound pressure was determined with a Bruel and Kjaer 0.5 in. $(1.25 \mathrm{~cm})$ condenser microphone and General Radio wave analyzer.

Procedure. The animals were initially trained to press and hold the telegraph key in the presence of the light and to release it at the onset of a $4 \mathrm{kHz}$ tone. Details of the training procedures are described by Moody (1970) and Moody et al. (1976). Key release terminated the tone and light. Latency was measured (in $\mathrm{ms}$ ) from tone onset to key release. Responses with latencies of less than $1 \mathrm{~s}$ were reinforced with a pellet of food. Releases with longer latencies terminated the light and tone, but were not reinforced. Following an intertrial interval of $5 \mathrm{~s}$ without a response, the light was again presented. Responses during the intertrial interval caused a new 5 -s interval to be started. The interval from key press in the light to tone onset was randomly varied from 1-4 s. Key release before tone onset terminated the light and started a new intertrial interval. Sessions were concluded when 350 trials had been presented. The average time required was about $1 \mathrm{~h}$ during training and testing sessions.

During initial training, only a fairly intense tone was presented, but when behavior had stabilized in terms of low numbers of intertrial responses and key releases before tone onset, variation in stimulus intensity was introduced. Stimuli were selected from a predetermined set and presented in a quasirandom order. The highest sound pressure level (SPL) presented was $86 \mathrm{~dB}$ (re $0.0002 \mathrm{dyne} / \mathrm{cm}^{2}$ ) (dB SPL). During each session, the lowest intensity resulting in median latencies greater than $1 \mathrm{~s}$ was determined for each animal and the stimulus range was adjusted such that only a single sound pressure resulted in latencies over $1 \mathrm{~s}$. This procedure insured a reasonably constant density of reinforcement during all sessions.

Latencies were recorded separately at each of the sound pressures presented and functions relating latency to stimulus sound pressure were the primary data obtained. When these functions stabilized in terms of low intersession and intrasession variability, experimental intervention with ethanol was begun.

Ethanol Administration. Ethanol was administered only on days subsequent to sessions in which LI functions fell within the range determined during baseline observations. On these days, $40 \%$ ethanol $(w / v)$ made up with sterile distilled water was infused into the catheter at a rate not in excess of $2 \mathrm{ml} / \mathrm{min}$. Saline was then infused to flush all of the ethanol into the bloodstream. The session was begun immediately following saline infusion and was terminated after $2 \mathrm{~h}$ if the animal did not complete 350 trials. Doses were from $1.0-2.5 \mathrm{~g} / \mathrm{kg}$, tested in a random order. Each dose was tested at least two times in each monkey, with additional tests being run if session variability was high. Drug sessions were run every 7 days on the average and were separated by no fewer than 3 days. Several sessions were also run in which the level of the test stimulus was not varied from trial to trial, but remained at $65 \mathrm{~dB}$ SPL. These sessions lasted at least $3 \mathrm{~h}$ and were run both with and without ethanol to monitor changes in latency during the session.

Noise Exposures. Following determination of the effects of ethanol on LI functions, the animals were exposed to octave band noise for 1 or $2 \mathrm{~h}$ at $100 \mathrm{~dB}$ SPL. The octave band employed was centered at 
Fig. 1

A typical function relating response latency to the sound pressure level (SPL) of the test tone. The vertical bars show the interquartile ranges of the latencies at each sound pressure level and the inset shows the frequency distribution of latencies obtained at the $63 \mathrm{~dB}$ SPL stimulus. In this and subsequent latency functions, there was one additional stimulus which was below the lowest one shown and which resulted in median latencies greater than $1000 \mathrm{~ms}$. The slope of the line extending upward and to the left from the point at $13 \mathrm{~dB}$ SPL is that which would be required to connect that point with the one not shown

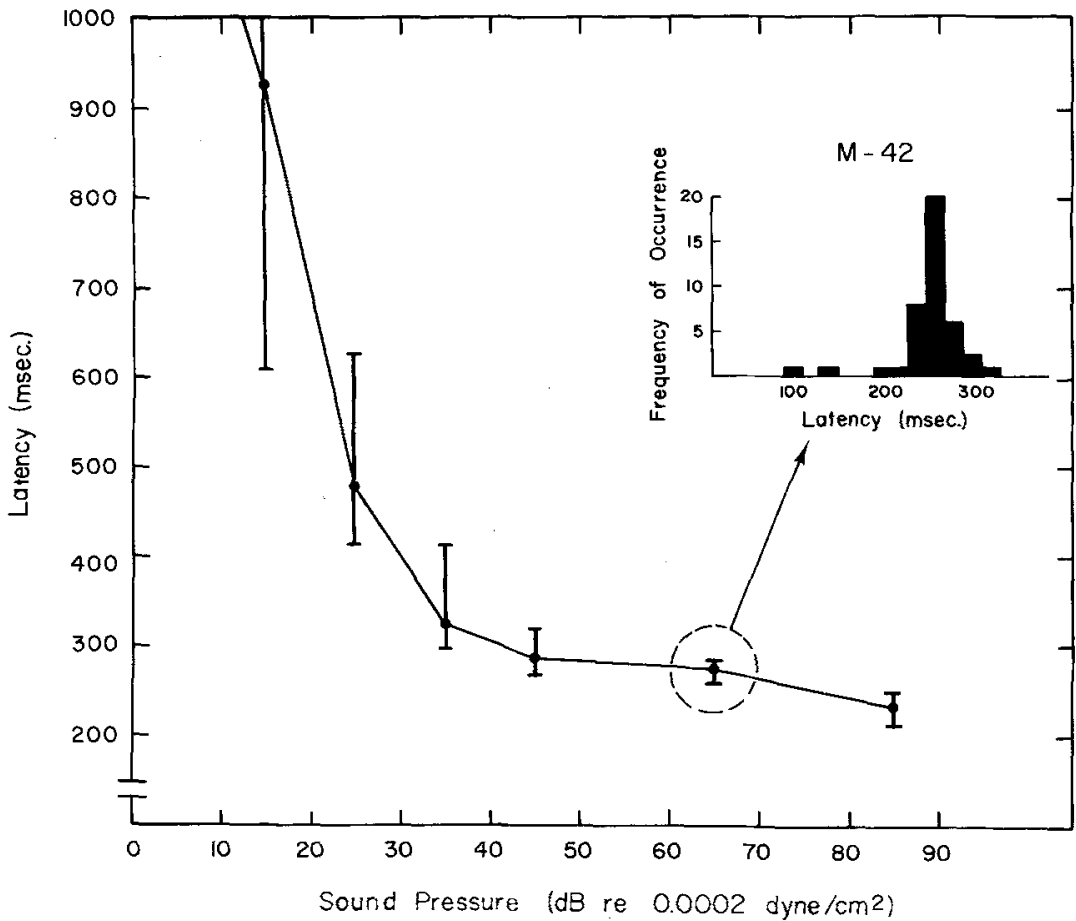

$2 \mathrm{kHz}$ and extended from $1.4-2.8 \mathrm{kHz}$. These exposures were chosen on the basis of previous data which indicated that they produced only temporary hearing deficits. Testing began within 5 min following the exposure and continued for about $1 \mathrm{~h}$.

\section{Results}

A representative LI function, along with a frequency distribution of latencies obtained at one stimulus level, is shown in Fig. 1. The decrease in median latency with increase in stimulus level is evident, as is the concomitant decrease in variability shown by the vertical bars connecting the quartile points. At higher stimulus levels, the interquartile range is generally found to be $20-30 \mathrm{~ms}$, but this value increases to several hundred milliseconds at low sound pressures. In subsequent figures, the quartile points have not been shown for reasons of clarity, but may be assumed to resemble those shown in this figure. The inset in the figure shows the frequency distribution of latencies to be relatively tight, with a mode centered at $250 \mathrm{~ms}$. The two short latency responses seen at 100 and $140 \mathrm{~ms}$ probably represent anticipatory responses, i.e., responses initiated before stimulus onset. Since there was no minimum latency criterion, these responses would have been reinforced.

Figure 2 presents the LI functions obtained from the three animals following ethanol $(1.0-2.5 \mathrm{~g} / \mathrm{kg})$. In this figure, and in similar figures which follow, the shaded area indicates the range of functions obtained on days immediately preceding ethanol administration and may be used as an indication of baseline latency values.

The figure shows clearly a dose-related increase in reaction time at the higher levels of stimulus in each of the three subjects. This ethanol-induced increase in reaction time is approximately equal at a given dose for all of the higher stimulus levels. At low levels, no increase is seen in the data for M41, but an increase which is generally comparable to that at the higher stimulus levels is seen for the remaining two subjects (M42 and M47).

At the lowest dose $(1.0 \mathrm{~g} / \mathrm{kg})$, there was essentially no effect and these data are assumed to serve as a vehicle-only control. At the highest dose $(2.5 \mathrm{~g} / \mathrm{kg})$ the behavior of all subjects was disrupted. M47 in particular tended to stop working on the testing procedure, and the median latencies shown in the figure for M47 are based on considerably fewer observations (about nine per stimulus) than are those obtained with other doses. This small sample size may account for the apparently normal latency at the highest stimulus level for M47 following $2.5 \mathrm{~g} / \mathrm{kg}$ ethanol. Both of the other monkeys continued to work when given $2.5 \mathrm{~g} / \mathrm{kg}$. M41, however, tended to make a large number of intertrial responses (i.e., responses when the light was off) during these sessions, thus reducing the number of tone presentations and the number of observations per median latency. M42 increased intertrial responses only slightly and did not significantly reduce the number of latencies which could be measured in the $2.5 \mathrm{~g} / \mathrm{kg}$ sessions. 

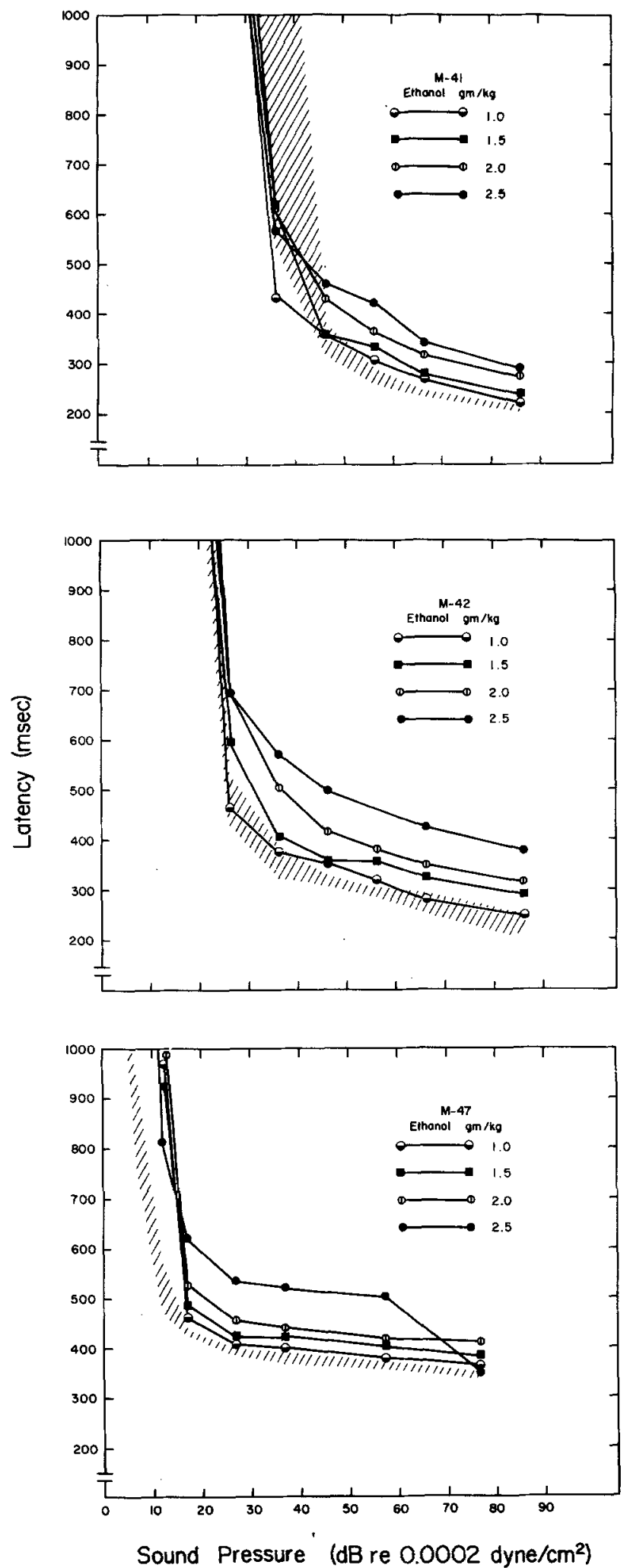

Fig. 2. Latency-intensity (LI) functions obtained from three animals following administration of various doses of ethanol. In each panel, the hatched area represents the range of latencies obtained on days immediately preceding the ethanol sessions. Each function represents the data for the last day run at a given dose

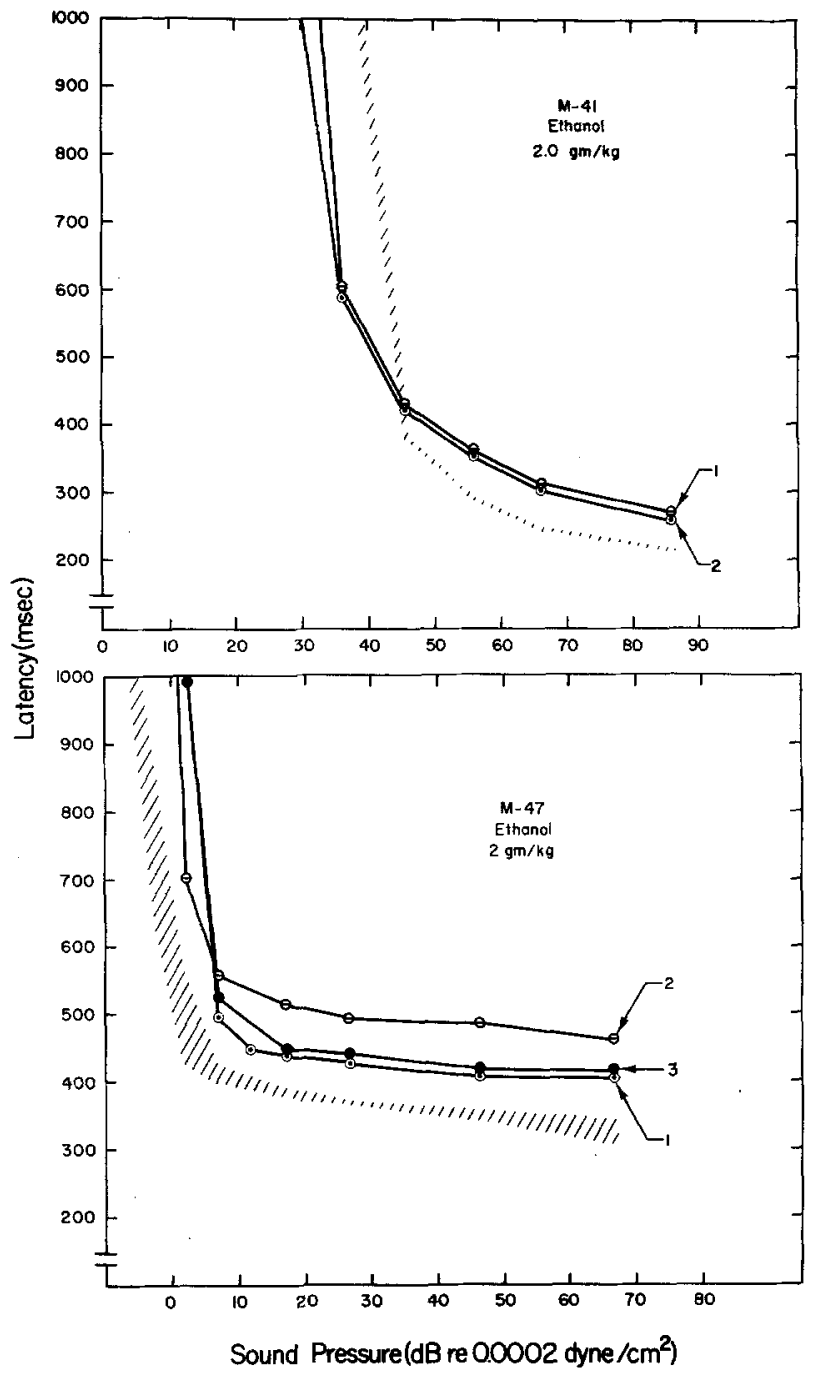

Fig. 3. Latency-intensity (LI) functions obtained from two animals in several sessions in which the ethanol dose was $2 \mathrm{~g} / \mathrm{kg}$. As in Figure 2, the hatched area represents the range of latencies obtained on days immediately preceding ethanol sessions

It is of interest to note that M41 actually showed a tendency toward increased sensitivity (shorter latencies) to low stimulus intensities following ethanol administration. This monkey had the highest sensory thresholds of the three subjects in the absence of ethanol, where threshold is taken as the stimulus level which resulted in a $1000 \mathrm{~ms}$ median latency (Pfingst et al., 1975b). In M47, however, which showed the lowest threshold, ethanol produced the greatest decrement in sensitivity (increased latencies) to low level stimuli. Monkey M42, intermediate in his ability to hear low level tones, showed a similar effect of a given dose of ethanol at all stimulus levels including the lowest.

The effects of ethanol on responding to low level stimuli as a function of baseline sensitivity can be seen in Fig. 3 as well for M41 and M47. In this figure, 
Fig. 4

A Means over six sessions of median latencies recorded in blocks of 20 trials following administration of $2 \mathrm{~g} / \mathrm{kg}$ ethanol (upper function) and from the six nondrug sessions immediately preceding those on which ethanol was administered (lower function). The stimulus level was $65 \mathrm{~dB}$ SPL. The vertical bars represent the interquartile ranges and have been displaced slightly from the data points for clarity. B Frequency distribution of latencies pooled over the six sessions entered into the means shown in panel A. C Frequency distribution of latencies pooled over the six control sessions shown in panel A

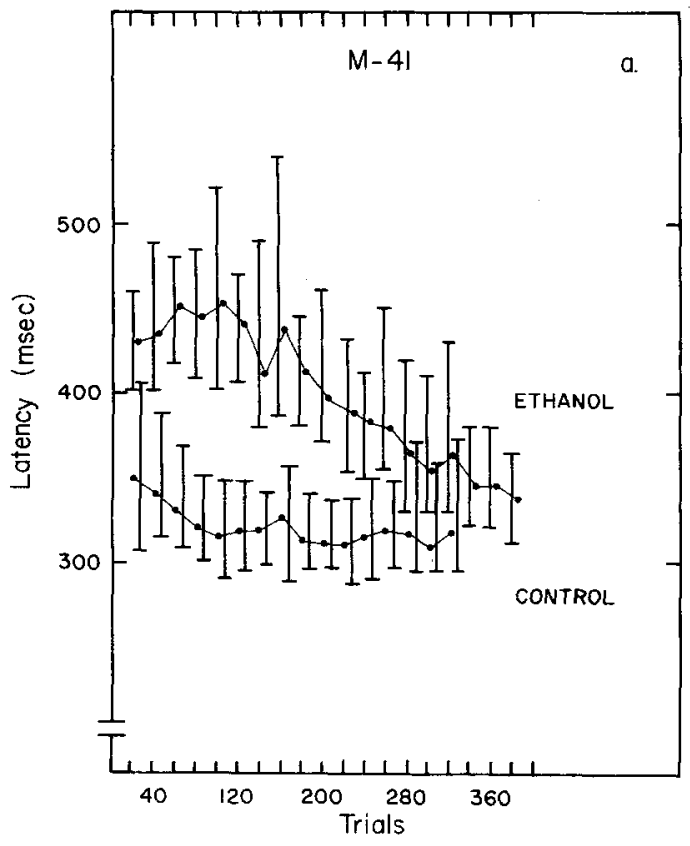

median latencies have been plotted for sessions in which $2.0 \mathrm{~g} / \mathrm{kg}$ was administered to demonstrate the repeatability of ethanol effects between sessions. For M41, the two ethanol sessions, separated by 9 days without drug administration, may essentially be superimposed. Note that although there is the expected increase in latencies at the high stimulus levels, there is an apparent decrease at the lowest levels. This effect is also seen in Fig. 2 for this monkey. In the data from M47, there is a much larger increase in median latencies in session two than in sessions one and three, which show about the same amount of increase. During session two, this animal stopped working frequently and completed only about half the normal number of trials. There was no apparent reason for the latency increase or the refusal to work. Session two was obtained 2 weeks after session one, and session three was obtained approximately 8 weeks after session two. Sessions at other dose levels occurred between the $2 \mathrm{~g} / \mathrm{kg}$ sessions. As is also seen in Fig. 2, the amount of the latency increase at a given dose for stimulus levels greater than $10 \mathrm{~dB}$ above threshold was essentially independent of the level of the stimulus. The replicability of LI functions for a given dose does not suggest the development of tolerance under these testing conditions.

Figure $4 \mathrm{a}$ presents median latencies across trials following administration of $2 \mathrm{~g} / \mathrm{kg}$ ethanol to M41. The data shown are means of the median latencies recorded in 20-trial blocks throughout six sessions in which the stimulus level was held constant at $65 \mathrm{~dB}$ SPL. The length of each of these sessions was at least $3 \mathrm{~h}$ for the ethanol condition. Control data have also been plotted for comparison, and consist of means of median latencies from the sessions immediately preceding the drug treatment days. The ethanol data show an initial increase in latency during trials $1-100$ followed by a decrease to near control latency values. The control data show a slight initial decrease in median latency followed by relatively constant values after about trial 80 . Figures $4 \mathrm{~b}$ and $4 \mathrm{c}$ present the latency distributions for single ethanol and control sessions, respectively. The control session produced a typical skewed latency distribution with a mode at $300 \mathrm{~ms}$, while the distribution from the ethanol session has a mode at $380 \mathrm{~ms}$ and is relatively broad, probably reflecting the progressive reduction in latency during the test session.

The relatively uniform effect of ethanol across stimulus levels may be contrasted with the effect shown in Fig. 5 of exposing subjects to moderately intense noise for 1 or $2 \mathrm{~h}$. Under this condition, there is no shift whatever in median latencies recorded at higher stimulus levels but there is a substantial shift at low levels. The significance of this effect, which reflects a sensory phenomenon called loudness recruitment, has been discussed in detail elsewhere (Moody, 1973). Briefly, the loudness recruitment phenomenon occurs when hearing losses are of cochlear (inner ear) origin, as in loss induced by noise or drugs. An increase in threshold is shown by the shift to the right in the upper limbs of the LI functions. Above threshold, however, the perceived loudness of the test tone increases rapidly until a point is reached at which the loudness of a given tone in the impaired ear is equal to the loudness that tone 

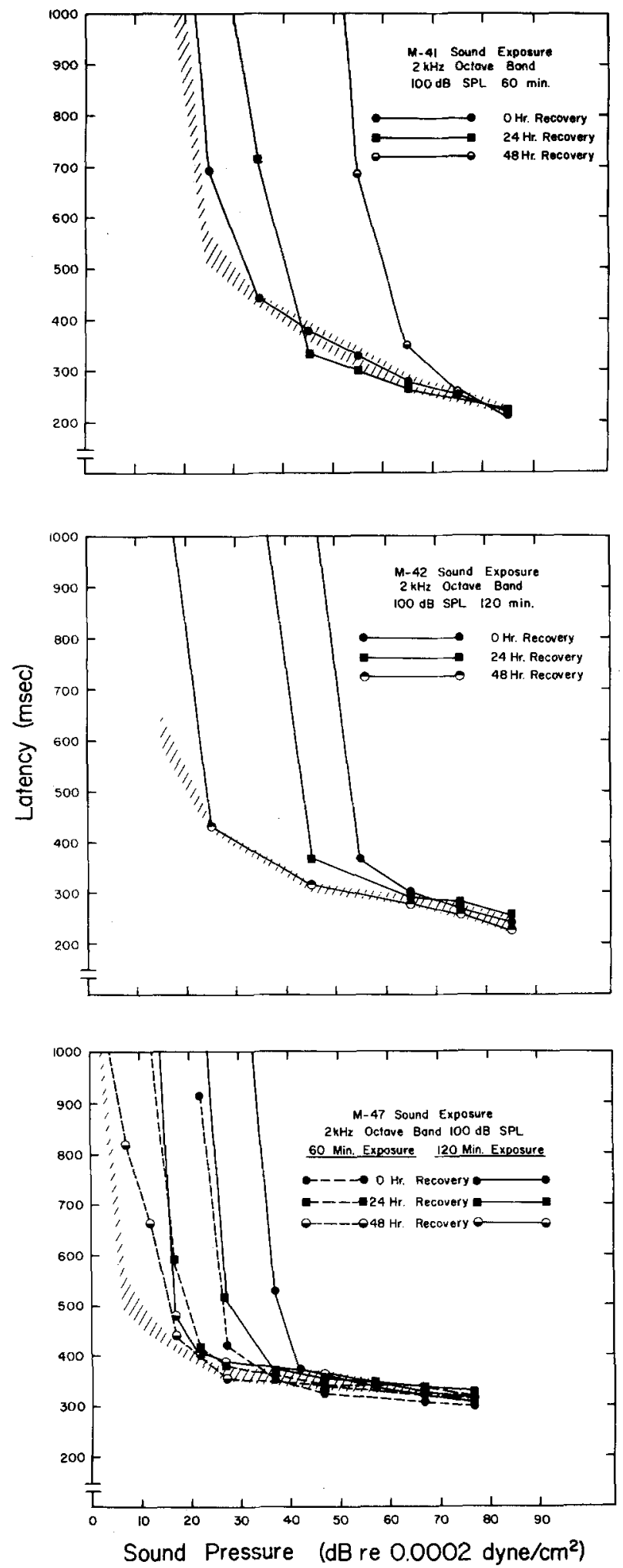

Fig. 5. Latency-intensity (LI) functions obtained from three animals following exposure to a $2 \mathrm{kHz}$ octave band of noise at $100 \mathrm{~dB} \mathrm{SPL}$ for the durations indicated on the figure. Functions are also shown at various intervals following exposure to illustrate the slow recovery. The hatched area represents the range of LI functions on days immediately preceding the exposures would have in the normal ear. The stimulus level at which this loudness equivalence occurs is that level at which the LI function, obtained following exposure, intersects the range of normal LI functions (the shaded area of Fig.5). It should also be noted that recovery from the effects of noise exposure takes place over at least $48 \mathrm{~h}$, and that there is considerable intersubject variability in the magnitude of effect resulting from a given noise exposure. These findings are both typical of noise-induced hearing loss.

\section{Discussion}

The data presented here confirm earlier reports that ethanol increases simple reaction time and augment previous results by demonstrating that, over a wide range of test tone levels, the amount of the increase is nondifferential with respect to stimulus level. That is, unlike noise exposure, ethanol does not increase reaction time to low level stimulation to any greater extent than it increases reaction time to higher level stimuli. In fact, in one of the three monkeys tested, ethanol did not significantly increase reaction time to a threshold-level tone even at doses that slowed reaction time to higher level tones. When compared to the effects of exposure to intense noise, which produces a marked increase in response latency at the lower stimulus levels, it is clear that ethanol does not alter the ability to hear low level stimuli. Rather, its major effect appears to be to produce a dose-related increment in the latency to response following tone presentation. This effect could be due either to an impairment of central processing of the incoming stimuli or to an impairment of the ability to make the response. This experiment does not allow a differentiation of these possibilities.

There is some indication that the ability of ethanol to alter the speed of response to low level stimuli depends on the baseline sensitivity of the monkeys. Thus, the monkey with the lowest threshold (i.e., the most sensitive) showed a loss in sensitivity following ethanol administration. This reduction in sensitivity was not dose-related, but occurred at all doses. On the other hand, the monkey with the highest threshold showed a tendency toward increased sensitivity (i.e., lower thresholds) following ethanol. The monkey with intermediate thresholds showed no sensitivity shift. This effect is reminiscent of the rate-dependent effects of drugs originally described by Dews (1958). Here, the effect of a drug on the latency seems to depend on the baseline latency of response to low level stimuli. Further observations of animals in tests of sensory acuity will be necessary to determine the generality of this finding. 
In conclusion, it seems clear from these results that ethanol, even in high doses, does not impair the ability of monkeys to hear auditory stimuli.

Acknowledgements. This research was supported by grants from NINCDS and NIDA.

\section{References}

Carpenter, J. A. : Effects of alcohol on some psychological processes. Q. J. Stud. Alcohol 23, 274-314 (1962)

Chocholle, R.: Variations des temps de reaction auditifs en fonction de l'intensite a diverse frequences. Annee Psychol. 41, 65-124 (1940)

Deneau, G. A., Yanagita, T., Seevers, M. H.: Self-administration of psychoactive substances by the monkey: A measure of psychological dependence. Psychopharmacologia 16, 30-48 (1969)

Dews, P. B. : Studies on behavior. Stimulant actions of methamphetamine. J. Pharmacol. Exp. Ther. 122, $137-147$ (1958)

Moody, D. B.: Equal brightness functions for supra-threshold stimuli in the pigmented rat. Vision Res. 9, 1381-1389 (1969)

Moody, D. B.: Reaction time as an index of sensory function in animals. In: Animal psychophysics: The design and conduct of sensory experiments, W. C. Stebbins, ed., pp. 277-301. New York: Appleton Century Crofts 1970

Moody, D. B.: Behavioral studies of noise-induced hearing loss in primates: Loudness recruitment. Adv. Otolaryngol. 20, 82-101 (1973)

Moody, D. B., Beecher, M. D., Stebbins, W. C.: Behavioral methods in auditory research. In: Handbook of auditory and vestibular research methods, C. A. Smith, J. A. Vernon, eds., pp. 439-497. Springfield: Charles C. Thomas 1976

Moody, D. B., Stebbins, W. C., Miller, J. M.: A primate restraint and handling system for auditory research. Behav. Res. Methods Instrum. 2, 180-182 (1970)

Moskowitz, H., Burns, M.: Effects of rate of drinking on human performance. Q. J. Stud. Alcohol 37, 598-605 (1976)

Moskowitz, H., Roth, S.: Effect of alcohol on response latency in object naming. Q. J. Stud. Alcohol 32,969-975 (1971)

Pfingst, B. E., Hienz, R., Kimm, J., Miller, J.: Reaction time procedure for measurement of hearing. Suprathreshold functions. J. Acoust. Soc. Am. 57, 421-430 (1975a)

Pfingst, B. E., Hienz, R., Miller, J.: Reaction-time procedure for measurement of hearing. Threshold functions. J. Acoust. Soc. Am. 57, 431-436 (1975b)

Pollack, J. D.: Reaction times to different wavelengths at various luminances. Percept. Psychophys. 3, 17-24 (1968)

Stebbins, W. C.: Auditory reaction time and the derivation of equalloudness contours for the monkey. J. Exp. Anal. Behav. 9, 135$142(1966)$

Tharp, V. K., Jr., Russell, O. H., Jr., Lester, B. K., Williams, H. L.: Alcohol and information processing. Psychopharmacologia 40, $33-52$ (1974)

Zunder, P. M.: Effects of alcohol and prediction outcome on extrafoveal signal detection. Q. J. Stud. Alcohol 38, 392-402 (1977)

Received August 3, 1979; Final Version January 7, 1980 